\title{
A WSRT Search for Millisecond Pulsars
}

\author{
W. W. Tian ${ }^{1,2}$, R. G. Strom ${ }^{3,4}$, B. W. Stappers ${ }^{4}$, X. Z. Zhang ${ }^{1}$,
} $\mathrm{X} . \mathrm{J} . \mathrm{Wu}^{5}$ and R. Ramachandran ${ }^{3,4}$

${ }^{1}$ Beijing Astronomical Observatory and Beijing Astrophysics Center of the National Astronomical Observatories, CAS, China

${ }^{2}$ Max-Planck-Institut für Radioastronomie, D-53121 Bonn, Germany

${ }^{3}$ NFRA, Postbus 2, 7990 AA Dwingeloo, Netherlands

${ }^{4}$ Astronomical Institute, University of Amsterdam, Netherlands

${ }^{5}$ Department of Geophysics, Beijing University, 100087, China

\begin{abstract}
Based on our examination of the characteristics of about 280 known pulsars and 40 millisecond pulsars (MSPs), we have derived selection criteria for MSP candidates: steep spectrum, highly linearly polarized point sources. The first sample of 14 candidates from the NVSS at $1.4 \mathrm{GHz}$ and the WENSS at $325 \mathrm{MHz}$ has been observed by the WSRT using PuMa at both 92 and $21 \mathrm{~cm}$ in May 1999. We have finished processing all data and find no evidence for MSPs for a dispersion measure range of $0-200 \mathrm{~cm}^{-3} \mathrm{pc}$ in this first sample.
\end{abstract}

There are many point-like sources which have not been identified in the NRAO VLA SKY Survey (NVSS) and Westerbork Northern Sky Survey (WENSS) catalogues released two years ago. Some of them could be pulsars (Han and Tian, 1999). We have therefore investigated the characteristics of known normal pulsars and MSPs. Figure 1 shows two properties of 280 normal pulsars with known linear polarization and flux density. The MSPs are somewhat more strongly polarized and have steeper spectra than normal pulsars (Tian et al. 2000).

Based on our study, we have selected a sample of candidate MSPs from the NVSS and WENSS catalogues according to the following criteria: steep spectrum $(\alpha \leq-1.5)$, high linear polarization $(\geq 15 \%)$, low flux density $(\leq 100$ mJy at $325 \mathrm{M} H z)$, and high galactic latitude $\left(\geq 30^{\circ}\right)$. Our selection criteria can distinguish MSP candidates from both radio quasars and compact steep spectrum sources.

Table 1 shows the parameters for 14 candidates which have been observed by the WSRT at both 92 and $21 \mathrm{~cm}$ in combination with the new pulsar machine PuMa. We observed each candidate source for about 215 seconds, corresponding to a $2 \mathrm{Mpt}$ time series with a sampling time of $0.1 \mathrm{~ms}$ at both frequencies. At $92 \mathrm{~cm}$ we used $39 \mathrm{kHz}$ channels and a $10 \mathrm{MHz}$ bandwidth, while at $21 \mathrm{~cm}$ we used $156 \mathrm{kHz}$ channels and an $80 \mathrm{MHz}$ bandwidth. This is sufficient to detect each of them if the emission is $100 \%$ pulsed. Including the time for set up and calibration, the total observation time was 3.5 hours. The data analysis was done using software developed by the pulsar group in Amsterdam.

With the data processing complete, the signal-to-noise ratio of most candidate pulsations over the range searched in period and $D M$ is under 5 , too low for any to be considered genuine new MSPs. Since our observations would have 

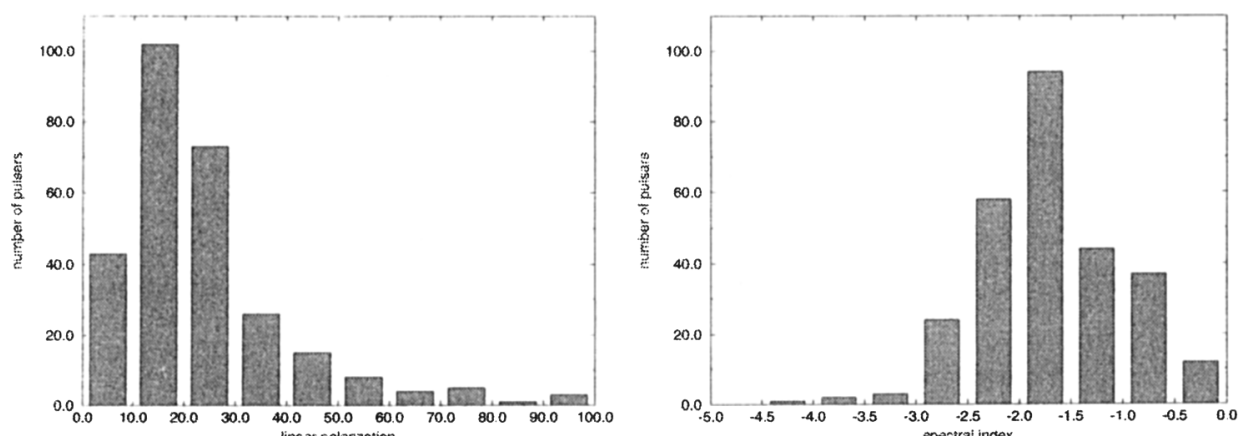

Figure 1. Distribution of degree of linear polarization and spectral index for 280 pulsars

been able to detect MSPs with $D M \leq 200 \mathrm{~cm}^{-3} \mathrm{pc}$, future searches should aim for distant MSP candidates with generally larger $D M$ values than in the present search. From our analysis of the data on all candidates with $D M \leq 200 \mathrm{~cm}^{-3} \mathrm{pc}$, we find no direct evidence that any of the sources is a MSP.

Table 1. Parameters of 14 MSP candidates from WENSS and NVSS

\begin{tabular}{|c|c|c|c|c|c|c|c|}
\hline $\mathrm{RA}(2000)$ & Dec (2000) & $S_{\mathrm{WI}}$ & $S_{\text {NVSS }}$ & $\omega$ & 1 & $\mathrm{~b}$ & $\mathrm{~L} / \mathrm{S}$ \\
\hline $\mathrm{h} \mathrm{m} s$ & & $\mathrm{Jy}$ & Jy & $\left(S \propto \nu^{\alpha}\right)$ & & & \\
\hline $\begin{array}{llll} & 376.0 & 16\end{array}$ & $\begin{array}{llll}55 & 28 & 13.4\end{array}$ & 0.036 & 0.0031 & -1.69 & 129.479 & 37.448 & 75 \\
\hline 160131.8 & $\begin{array}{lll}33 & 30 & 24.7\end{array}$ & 0.027 & 0.0024 & -1.66 & 20.654 & 48.434 & 71 \\
\hline 090502.6 & 495301.7 & 0.028 & 0.0028 & -1.58 & 136.149 & 42.217 & 44 \\
\hline 083527.9 & $\begin{array}{llll}66 & 58 & 49.1\end{array}$ & 0.030 & 0.0032 & -1.54 & 115.399 & 35.387 & 42 \\
\hline 095431.3 & 473042.7 & 0.034 & 0.0027 & -1.74 & 137.320 & 50.663 & 31 \\
\hline 112442.3 & $\begin{array}{lll}38 & 31 & 40.8\end{array}$ & 0.024 & 0.0022 & -1.64 & 142.341 & 69.188 & 27 \\
\hline 161427.5 & 343750.7 & 0.028 & 0.0030 & -1.54 & 22.639 & 45.859 & 27 \\
\hline 091255.3 & $\begin{array}{lll}57 & 39 & 40.7\end{array}$ & 0.020 & 0.0021 & -1.55 & 125.570 & 41.903 & 22 \\
\hline 134640.4 & $\begin{array}{llll}60 & 03 & 17.2\end{array}$ & 0.041 & 0.0032 & -1.75 & 77.192 & 55.887 & 21 \\
\hline 152654.7 & $\begin{array}{lll}60 & 58 & 50.7\end{array}$ & 0.047 & 0.0038 & -1.73 & 62.952 & 47.292 & 21 \\
\hline $0840 \quad 07.7$ & 322552.3 & 0.021 & 0.0021 & -1.58 & 158.439 & 36.590 & 19 \\
\hline 112146.6 & 414704.2 & 0.032 & 0.0036 & -1.50 & 135.411 & 67.116 & 19 \\
\hline 122947.3 & $\begin{array}{lll}37 & 25 & 43.8\end{array}$ & 0.046 & 0.0034 & -1.79 & 110.758 & 79.188 & 19 \\
\hline $\begin{array}{lll}09 & 16 & 15.0\end{array}$ & 464428.1 & 0.028 & 0.0026 & -1.63 & 140.167 & 44.401 & 15 \\
\hline
\end{tabular}

\section{References}

Han, J.L. \& Tian, W.W. 1999, A\&AS, 136, 571

Tian, W.W., Strom, R.G., Stappers, B.W., Zhang, X.Z., Wu, X.J., Ramachandran, R., 2000, Proceedings of IAU Colloquium 177, eds: Kramer, M. et al., in press. 